\title{
Low Incidence of Anti-CD36 Antibodies in Acquired Thrombotic Thrombocytopenic Purpura
}

Silvia Ferrari, Verena Schitt, Hanspeter Rottensteiner* and Friedrich Scheiflinger

Global Biologics R \& D, Baxter Innovations $\mathrm{GmbH}$, Vienna, Austria

\begin{abstract}
Acquired idiopathic thrombotic thrombocytopenic purpura (TTP) is characterized by severe ADAMTS13 deficiency due to the presence of autoantibodies against ADAMTS13. Previous studies using Western blot and immunoprecipitation techniques detected autoantibodies against the membrane antigen CD36 in over $50 \%$ of acquired TTP patients. The pathological or clinical significance of this apparent correlation however remains elusive. We reevaluated this potential link by analyzing a cohort of 76 patients with acute acquired TTP for the presence of anti-CD36 autoantibodies using a newly developed enzyme-linked immunosorbent assay (ELISA). Circulating anti-CD36 antibodies were detected in only 4/76TTP patients and 2/63 healthy donors, with no statistically significant difference $(p=0.096)$ in antibody levels between the two populations. The gross discrepancy in the frequency of anti-CD36 antibodies detected in previous studies and the current one is likely due to the difference in methods used for antibody detection and in sample size. Our data indicate that patients with acquired TTP do not exhibit an increased incidence of anti-CD36 antibodies.
\end{abstract}

Keywords: Thrombotic thrombocytopenic purpura; ADAMTS13; CD36; Anti-CD36 antibodies

Thrombotic thrombocytopenic purpura (TTP) is a life-threatening thrombotic microangiopathy characterized by microangiopathic hemolytic anemia, thrombocytopenia with renal impairment or neurological abnormalities [1]. It is mainly caused by a functional deficiency of ADAMTS13 (adisintegrin and metalloproteinase with thrombo spondin motifs member 13), with development of inhibiting autoantibodies causing the acquired form of TTP [2,3]. ADAMTS13 is a plasma enzyme responsible for the cleavage of unusually large (UL) von Willebrand factor (VWF) multimers. Conversion of hyperactive ULVWF multimers to smaller and less active forms prevents microvascular platelet aggregation and thrombus formation.

The CD36 antigen (originally identified in platelets as glycoprotein IV) is an integral membrane protein of 471 amino acids belonging to the class B scavenger receptor family. CD36 is expressed mainly in professional phagocytes, platelets, microvascular endothelium and fat and muscle cells [4]. CD36 has multiple physiologic ligands including oxidized low-density lipoprotein, phosphatidyl serine and oxidized phosphatidyl serine expressed on the surface of apoptotic cells, longchain fatty acids and the type I repeats on thrombospondin 1 [4]. CD36 was also demonstrated to bind to ADAMTS13 in vitro [5], suggesting a functional link between the two proteins. Early reports have described the presence of autoantibodies directed against CD36 in about 50$70 \%$ of TTP patients [6-8]. Rock et al. [9] reported the concomitant presence of anti-CD36 and anti-ADAMTS13 antibodies in 16/35 TTP patients. These studies suggest that anti-CD36 antibodies might play a pathogenic role in acquired TTP, similar to the situation in patients with autoimmune diseases such as systemic lupus erithematosus [10] and antiphospholipid syndrome [11], where CD36 autoantibodies were detected.

Given that these early reports used methods with relatively low sensitivity and/or specificity, we reevaluated the potential link between the presence of anti-CD36 antibodies and acquired TTP. For this purpose, we developed a sensitive ELISA-based assay using human recombinant full-length CD36 for coating and a biotin-conjugated goat anti-human IgG antibody for detection of bound anti-CD36 antibodies. The cut-off for the assay was established by measuring the optical densities (OD) of plasma from 63 healthy donors. Ratios of sample OD to background OD (signal derived from pooled normal human plasma $[\mathrm{NHP}]$ ) were calculated and normal distribution assessed by Shapiro-Wilk test after log-transforming the data. Means and standard deviations of the resulting 63 ratios were calculated and the thresholds obtained by back-transforming the means plus three standard deviations of the transformed data. Samples with ratios below or above the cut-off levels were judged as negative or positive, respectively. As human-derived positive controls are not available, we used a monoclonal rat anti-human CD36 antibody directed against the extracellular part of human CD36 (R\&D Systems, Abingdon, United Kingdom) together with HRP-conjugated rabbit anti-rat IgG (Sigma, St. Louis, MO, USA)to monitor assay performance.

The presence of anti-CD36 antibodies was investigated in 76 patients diagnosed with acute acquired idiopathic TTP in samples taken prior to any therapy. Clinical characteristics and laboratory findings of these patients have previously been described $[12,13]$. Diagnosis of acute TTP was based on the following criteria: (i) presence of microangiopathic hemolytic anemia (hemoglobin level $<12 \mathrm{~g} \mathrm{dL}^{-1}$ ), direct antiglobulin test negative, at least two schistocytes per high-power field in the peripheral blood smear, increased lactate dehydrogenase levels, and undetectable serum haptoglobin; (ii) thrombocytopenia; and (iii) severely reduced $(\leq 10 \%)$ plasma ADAMTS13 activity levels. Fever, neurological symptoms or renal failure were not mandatory.

Circulating anti-CD36 antibodies were detected in 2/63 (3\%) healthy donors and in only 4/76 (5.2\%) TTP patients (Figure 1). All but

*Corresponding author: Hanspeter Rottensteiner, Global Biologics R \& D, Baxter Innovations GmbH, Industriestrasse 67, 1221 Vienna, Austria, Tel: +43 120100 2472941; Fax: +43 120100 5046; E-mail: Hanspeter_Rottensteiner@baxter.com

Received June 18, 2013; Accepted July 15, 2013; Published July 18, 2013

Citation: Ferrari S, Schitt V, Rottensteiner H, Scheiflinger F (2013) Low Incidence of Anti-CD36 Antibodies in Acquired Thrombotic Thrombocytopenic Purpura. J Blood Disorders Transf 4:146. doi:10.4172/2155-9864.1000146

Copyright: ( 2013 Ferrari S, et al. This is an open-access article distributed unde the terms of the Creative Commons Attribution License, which permits unrestricted use, distribution, and reproduction in any medium, provided the original author and source are credited. 
Citation: Ferrari S, Schitt V, Rottensteiner H, Scheiflinger F (2013) Low Incidence of Anti-CD36 Antibodies in Acquired Thrombotic Thrombocytopenic Purpura. J Blood Disorders Transf 4:146. doi:10.4172/2155-9864.1000146

one patient tested positive for anti-CD36 antibodies had undetectable ADAMTS13 activity that was associated with functional inhibitory IgG anti-ADAMTS13 antibodies.

Evaluation of the data using Mann-Whitney rank sum test (SigmaPlot; Systat Software, San Jose, CA)revealed no statistically significant difference $(p<0.096)$ in anti-CD36 antibody levels between healthy donors and TTP patients (Figure 1). Thus, our results do not confirm previous observations that about $50-70 \%$ of TTP patients have autoantibodies against CD36 [6-8]. The gross discrepancy is likely due to the difference in methods used for antibody detection. We performed an ELISA using recombinant CD36 as protein source and the biotinstreptavidin system for detection which is well-known to confer high sensitivity. In the past, sources of human CD36 were crude platelet lysates or CD36-enriched fractions thereof and the presence of antiCD36 antibodies was evaluated by Western blot with patient plasma as first antibody or by immunoprecipitation of patient plasma antibodies with CD36 derived from platelet lysates. This may have led to a high incidence of false positives due to binding of antibodies to unrelated proteins. Furthermore, we tested 76 TTP plasma samples, whereas

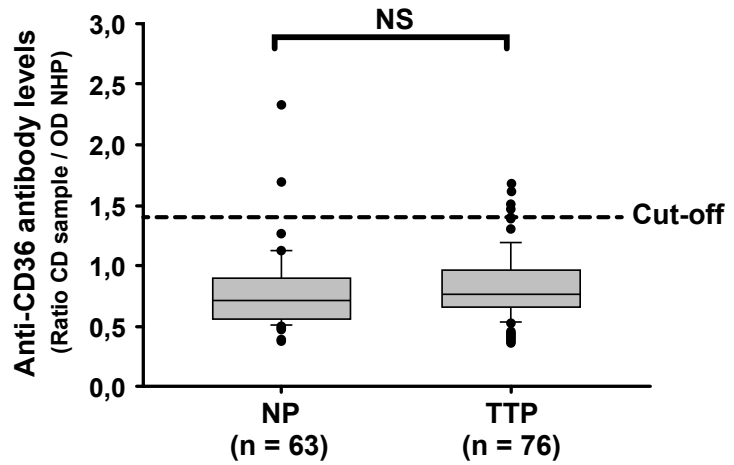

Microtiter plates (Costar, Corning Inc., NY, USA) were coated with recombinant human full-length CD36 expressed in human embryonic kidney 293 cells(rCD36; Origene, Rockville, USA) at $1.5 \mu \mathrm{g} / \mathrm{mL}$ in $0.05 \mathrm{M}$ carbonate-bicarbonate buffer, pH 9.6 (Sigma, St. Louis, MO, USA) by overnight incubation at $4^{\circ} \mathrm{C}$. Non-specific binding sites were blocked with Protein-Free blocking buffer (Thermo Fischer, Rockland, IL, USA). Plasma samples from 76TTP patients and 63 healthy donors (diluted 1:50 in LowCross buffer; Candor Bioscience, Weissensberg, Germany) were then added and incubated for $3 \mathrm{~h}$ at RT. Plates were washed and incubated with a biotin-conjugated goat anti-human IgG antibody (Rockland Immunochemicals, Gilbersville, PA, USA) followed by addition of streptavidinperoxidase polymer conjugate (Sigma). Anti-CD36 antibodylevels are presented as a ratio between the OD of the sample and that of a pooled NHP. The dashed line represents the cut-off of the assay (1.45). The bottom, median and top lines of the box mark the $25^{\text {th }}, 50^{\text {th }}$, and $75^{\text {th }}$ percentiles, respectively. The vertical line shows the range of values between the $5^{\text {th }}$ and $95^{\text {th }}$ percentiles; the dots represent outlier values. P-values $<0.05$ were considered statistically significant. NS= non-significant statistical difference between both groups .

Figure 1: Detection of anti-CD36 antibodies in patients with acquired TTP previous studies used only 12 to 35 patients [6-9]. Such differences in sample size could also introduce bias in the observed frequency of positive samples.

In conclusion, we are confident that our data show that the wellestablished presence of anti-ADAMTS13 antibodies in patients with acquired TTP is not accompanied by a higher incidence of circulating anti-CD36 antibodies than in healthy individuals.

\section{Acknowledgments}

The authors are grateful to Dr. Johanna A. Kremer Hovinga (University Hospita and University of Berne, Berne, Switzerland), Dr. Paul Knöbl (Medical University of Vienna, Vienna, Austria) and Agnès Veyradier (Hospital Antoine Béclère and ParisSud School of Medicine, Paris, France) for providing patient plasma samples and clinical information. The study was approved by the responsible ethic committees according to the Declaration of Helsinki. We are indebted to Karima Benamara for expert editing of the manuscript.

\section{References}

1. Moake JL (2002) Thrombotic microangiopathies. N Engl J Med 347: 589-600.

2. Tsai HM, Lian EC (1998) Antibodies to von Willebrand factor-cleaving protease in acute thrombotic thrombocytopenic purpura. N Engl J Med 339: 1585-1594.

3. Furlan M, Robles R, Galbusera M, Remuzzi G, Kyrle PA, et al. (1998) von Willebrand factor-cleaving protease in thrombotic thrombocytopenic purpura and the hemolytic-uremic syndrome. N Engl J Med 339: 1578-1584.

4. Silverstein RL, Febbraio M (2009) CD36, a scavenger receptor involved in immunity, metabolism, angiogenesis, and behavior. Sci Signal 2: re3.

5. Davis AK, Makar RS, Stowell CP, Kuter DJ, Dzik WH (2009) ADAMTS13 binds to CD36: a potential mechanism for platelet and endothelial localization of ADAMTS13. Transfusion 49: 206-213.

6. Tandon NN, Rock G, Jamieson GA (1984) Anti-CD36 antibodies in thrombotic thrombocytopenic purpura. Br J Haematol 88: 816-825.

7. Schultz DR, Arnold PI, Jy W, Valant PA, Gruber J, et al. (1998) Anti-CD36 autoantibodies in thrombotic thrombocytopenic purpura and other thrombotic disorders: identification of an $85 \mathrm{kD}$ form of $\mathrm{CD} 36$ as a target antigen. $\mathrm{Br} J$ Haematol 103: 849-857.

8. Wright JF, Wang H, Hornstein A, Hogarth M, Mody M, et al. (1999) Characterization of platelet glycoproteins and platelet/endothelial cell antibodies in patients with thrombotic thrombocytopenic purpura. Br J Haematol 107: 546-555.

9. Rock G, Anderson D, Clark W, Leblond P, Palmer D, et al. (2005) Does cryosupernatant plasma improve outcome in thrombotic thrombocytopenic purpura? No answer yet. Br J Haematol 129: 79-86.

10. Al-Shahi R, Mason JC, Rao R, Hurd C, Thompson EM, et al. (1997) Systemic lupus erythematosus, thrombocytopenia, microangiopathic haemolytic anaemia and anti-CD36 antibodies. Br J Rheumatol 36: 794-798.

11. Pelegri Y, Cerrato G, Martinuzzo ME, Carreras LO, Forastiero RR (2003) Link between anti-CD36 antibodies and thrombosis in the antiphospholipid syndrome. Clin Exp Rheumatol 21: 221-224.

12. Rieger M, Mannucci PM, Kremer Hovinga JA, Herzog A, Gerstenbauer G, et al (2005)ADAMTS13 autoantibodies in patients with thrombotic microangiopathies and other immunomediated diseases. Blood 106: 1262-1267.

13. Ferrari S, Mudde GC, Rieger M, Veyradier A, Kremer Hovinga JA, et al. (2009) IgG subclass distribution of anti-ADAMTS13 antibodies in patients with acquired thrombotic thrombocytopenic purpura. J Thromb Haemost 7: 1703-1710.
Citation: Ferrari S, Schitt V, Rottensteiner H, Scheiflinger F (2013) Low Incidence of Anti-CD36 Antibodies in Acquired Thrombotic Thrombocytopenic Purpura. J Blood Disorders Transf 4:146. doi:10.4172/2155-9864.1000146 